\title{
Relación entre diseño instruccional y rendimiento académico en un curso presencial y bimodal de Matemática: Un estudio cuasiexperimental
}

Espinoza Guzmán, Julia; Rodríguez Granados, Natalia; Moreira-Mora, Tania Elena

Relación entre diseño instruccional y rendimiento académico en un curso presencial y bimodal de Matemática: Un estudio cuasiexperimental

Revista Educación, vol. 42, núm. 2, 2018

Universidad de Costa Rica, Costa Rica

Disponible en: http://www.redalyc.org/articulo.oa?id=44055139035

DOI: https://doi.org/10.15517/revedu.v42i2.28763

Esta obra está bajo una Licencia Creative Commons Atribución-NoComercial-SinDerivar 3.0 Internacional. 


\title{
Relación entre diseño instruccional y rendimiento académico en un curso presencial y bimodal de Matemática: Un estudio cuasiexperimental
}

\author{
Relationship between instructional design and academic performance in a face -to face and blended Mathematics \\ course: A quasi experimental study
}

Julia Espinoza Guzmán [1]

DOI: https://doi.org/10.15517/revedu.v42i2.28763

Instituto Tecnológico de Costa Rica, Costa Rica

Redalyc: http://www.redalyc.org/articulo.oa?id=44055139035

juliaespinoza@itcr.ac.cr

Natalia Rodriguez Granados [2]

InstitutoTecnológico de Costa Rica, Costa Rica

nrodriguez@itcr.ac.cr

Tania Elena Moreira-Mora [3]

Instituto Tecnológico de Costa Rica, Costa Rica

tmoreira@itcr.ac.cr

(iD) http://orcid.org/0000-0002-8955-080

\section{RESUMEN:}

La finalidad de esta investigación consistió en analizar la relación del diseño instruccional (DI) de un curso de Cálculo y Álgebra Lineal (CAL) en modalidad presencial y bimodal con el rendimiento académico del estudiantado. El diseño del estudio fue cuasiexperimental y se ejecutó en dos fases, una de planificación y otra de intervención, en una muestra de 64 estudiantes del Instituto Tecnológico de Costa Rica. Los datos de las variables del diseño instruccional: objetivos, contenidos curriculares, actividades aprendizaje, materiales educativos y estrategias de evaluación fueron recolectados en el 2013 con una escala tipo Likert mediante la técnica de la encuesta autoadministrada. Según los resultados de la t de student y de regresión lineal múltiple, se comprobó que existe una relación estadísticamente significativa entre la percepción del estudiantado sobre el diseño instruccional y su rendimiento académico. Con estos hallazgos se evidencia que una adecuada planificación del curso contribuye positivamente con el desempeño académico del estudiantado, sea en una modalidad presencial o bimodal. Por ello la principal recomendación es incentivar a los docentes a usar el diseño instruccional en la organización de un curso y valorar como una alternativa la opción bimodal por su flexibilidad de horarios, sobre todo al comprobar que el tipo de modalidad no es un factor significativo en el rendimiento en Matemática.

Palabras Clave: Diseño Instruccional, Rendimiento académico, Matemática, Curso presencial, Curso bimodal, Cuasiexperimental.

\section{ABstract:}

The purpose of this research was to analyze the relationship between the instructional design of a face-to-face and blended Calculus and Linear Algebra (CAL) course with student performance. The design of the study was quasi-experimental with a sample of 64 students enrolled at Instituto Tecnológico de Costa Rica. The study was implemented in two phases: planning phase and intervention phase. The data of the instructional design variables: objectives, contents, learning activities, educational materials and assessment strategies were collected in 2013 using a Likert scale through a self-administered survey technique. Based on the results of the student' $s t$ and the multiple linear regression, it was found that there is a statistically significant relationship between the perception of the students regarding the instructional design and their performance. These findings indicate that an adequate

\section{NotAS DE AUTOR}

[1] Docente de la Escuela de Computación y coordina la capacitación en e-learning del TEC Digital. Es ingeniera en Computación del TEC y Licenciada en Educación de la UNED

[2] Docente de la Escuela de Matemática del TEC, graduada del Bachillerato y la Licenciatura en Enseñanza de la Matemática Asistida por Computadora del Instituto Tecnológico de Costa Rica y de la Maestría académica en Matemática Educativa de la Universidad de Costa Rica.

[3] Tiene un Doctorado en Educación de la UNED, una Maestría en Evaluación Educativa de la Universidad de Costa y una Licenciatura en Literatura y Lingüística de la Universidad Nacional. Labora en el Departamento de Orientación y Psicología, destacada en el Comité Examen de Admisión. 
planning in the design of a course contributes positively in the performance of the students, in either a face-to-face or a blended course. Therefore, the main recommendation is to encourage professors to use the instructional design in the organization of a course and the blended modality as an alternative due to its schedule flexibility, especially when proving that the type of modality is not a significant factor in the academic performance.

KEYWORDS: Instructional design, Academic achievement, Mathematics, face-to-face course, Blended course, Quasiexperimental.

\section{INTRODUCCIÓN}

Esta investigación se orientó hacia el análisis de la relación entre el diseño instruccional (DI) en el rendimiento académico entre dos grupos del mismo curso de Cálculo y Álgebra Lineal en modalidad presencial y bimodal del Instituto Tecnológico de Costa Rica (TEC), con la finalidad de responder a la siguiente interrogante: ¿Existe relación entre la planificación planteada en el diseño instruccional y el rendimiento académico del estudiantado en un curso de Cálculo y Álgebra Lineal? De acuerdo con Landa y Peñalosa (2009), el diseño instruccional puede tener la misma relevancia que otros factores como los contenidos curriculares, el profesorado y el alumnado en el rendimiento académico.

El diseño instruccional es un proceso sistémico para desarrollar las especificaciones de aprendizaje, que inicia la identificación de características de los estudiantes y de sus requerimientos de aprendizaje, incluye la especificación de objetivos, contenidos, selección de la propuesta metodológica para el aprendizaje, definición de materiales educativos y especificación de la propuesta evaluativa (Brown \& Green, 2015; Piskurich, 2015; Umaña, 2017). De acuerdo con Gil (2004, p. 94), planear consiste en "prever con claridad las metas que han de alcanzar para hacer realidad un proyecto e identificar los medios que permitan el logro de esas metas" y específicamente el plan de un curso a través del diseño instruccional es un esquema de lo que será el proceso de aprendizaje que "establece el dónde, el cuándo, el cómo y el con qué se llevará a cabo la instrucción, vista desde diferentes teorías: del aprendizaje, de sistemas, de la evaluación” (Gil, 2004 p. 95) y de la tecnología educativa. Entre las características fundamentales del DI como proceso están que:

- realiza un diagnóstico de la población meta, de sus necesidades educativas, de las características del modelo educativo y de la institución educativa,

- permite planificar y generar experiencias positivas (Arjona y Blando, 2007, p. 1) tanto para estudiantes como para profesores.

- la o el docente reflexiona sobre las teorías educativas, estrategias para el aprendizaje y la evaluación, para hacer una selección acorde con los hallazgos del diagnóstico,

- es un proceso integrado de elementos que interactúan entre sí (Gustafson \& Branch, 2002), que exige coherencia, enfocado en el estudiante, es un esfuerzo de un equipo interdisciplinario,

- se considera que es un "puente" o conexión entre la teoría del aprendizaje y la práctica educativa, dado que supone un cuerpo de conocimientos que prescribe acciones educativas para optimizar los resultados de aprendizaje deseados (Ertmer \& Newby, 2013; Reigeluth, 1983).

\section{ESTADO DE LA CUESTIÓN}

Actualmente existe una tendencia hacia el estudio de "variables de proceso de la acción instruccional para establecer su efecto en el rendimiento [académico], considerando que, diferentes contextos instruccionales, deben llevar consigo diversos procesos instruccionales y producir un rendimiento diferente" (De la Fuente, Martínez, Peralta \& García, 2010, p. 806-807). Los mismos autores recalcan que, se han realizado diversos estudios y que: "sin embargo, la interacción entre el nivel de rendimiento con los contextos instruccionales y la percepción del proceso de [enseñanza y aprendizaje] no ha sido suficientemente abordada” (p. 807). 
En cuanto a los estudios enfocados en esta relación entre el diseño instruccional y el rendimiento académico en educación superior, se destaca el trabajo de Venkatesh, Croteau \& Rabah (2014) realizado en 12 universidades de Quebec, Canadá, sobre cómo la eficacia percibida de un curso puede estar vinculada a las percepciones de los métodos instructivos de enseñanza empleados por el personal docente y los enfoques de aprendizaje adoptados por el alumnado. Se aplicó una encuesta a 14.283 estudiantes, con preguntas sobre tecnologías de información y comunicación (TIC), métodos de enseñanza, formas de conducir las clases del profesorado y el uso de trabajo en grupo y discusiones, así como del material empleado en clases. Para analizar la información recopilada utilizaron un Exploratory Factor Analysis (EFA) y modelos de regresión lineal. Concluyeron que la modalidad del curso (presencial, bimodal o virtual) no es un factor determinante para el aprendizaje, mientras que, en cuanto a calidad de sus cursos, más bien el alumnado enfatiza en la forma en que sus instructores eligen y preparan su material didáctico, la forma en que se entrega, las formas en que las instructoras y los instructores se ocupan de diferentes estilos de aprendizaje, y en los desafíos intelectuales que se presentan durante el curso.

Por otra parte, Landa \& Peñalosa (2009) realizaron una investigación en la cual destacaron la importancia del diseño instruccional en el proceso de aprendizaje. Su estudio fue de tipo factorial cuasiexperimental con 121 estudiantes de psicología, quienes fueron distribuidos en cuatro grupos, dos presenciales y dos en línea. En la investigación se analizó la efectividad de dos modelos instruccionales: uno de transmisión de conocimientos y otro de construcción del conocimiento; cada modelo se implementó en un grupo presencial y en uno en línea. Para probar la efectividad de los modelos, todos los grupos realizaron una prueba objetiva. Las pruebas estadísticas ejecutadas dieron como resultado que el modelo de construcción del conocimiento fue más efectivo que el de transmisión de conocimientos. Posteriormente, compararon los grupos donde se aplicó el modelo de construcción de conocimientos, para ver la efectividad de los cursos presenciales $(\mu=50.3)$ respecto a los cursos línea $(\mu=58.2)$; de donde se obtuvo que los últimos fueron más efectivos, pues las diferencias observadas fueron estadísticamente significativas.

De la Fuente et al. (2010) realizaron un estudio en España, donde analizaron la relación entre la percepción del proceso de enseñanza-aprendizaje y el rendimiento académico. El estudio se realizó con una muestra de 2020 estudiantes de las universidades de Almería y Granada, y utilizaron un diseño cuasiexperimental, con medidas post-test y correlacional. Se consideró el contexto y la percepción del proceso de enseñanza aprendizaje como variables independientes y el rendimiento como dependiente. Para medir la percepción del estudiantado se empleó una escala de evaluación de 30 ítems y para medir el rendimiento se consideró la puntuación media en una asignatura anual. Para el análisis de datos se utilizaron ANOVAs y MANOVAs entre las variables definidas; así como un análisis estructural. Entre los hallazgos que encontraron se puede mencionar que el estudiante o la estudiante con un alto rendimiento tiene una mejor percepción del proceso de enseñanza aprendizaje.

En otra investigación realizada en la Universidad de Granada con 1431 estudiantes ya en matrícula en varios cursos de estudios empresariales de primer año de pregrado entre 2008 y el 2010, López-Pérez, PérezLópez \& Rodríguez-Ariza (2011) se propusieron, por un lado, analizar el efecto de una experiencia de aprendizaje en ambientes bimodales en la tasa de abandono en los cursos y en los resultados de los exámenes finales y, por el otro, examinar el grado en que estas experiencias aprendizaje en ambientes bimodales se relacionaban con la percepción del alumnado (medida subjetiva) y la calificación final del curso (medida objetiva). A partir de los resultados encontraron que la inclusión de actividades en ambientes bimodales tiene un efecto positivo en la reducción de las tasas de abandono de los cursos e incrementó las tasas de aprobación de los exámenes de las materias, así como en las notas finales. Esto les permitió concluir que cuanto mayor sea la percepción del estudiantado respecto a las actividades de aprendizaje, mayor es su motivación y satisfacción.

En una investigación similar en México, Andrade-Aréchiga, López \& López-Morteo (2012) realizaron la evaluación de unas unidades de aprendizaje asociadas a conceptos matemáticos de un primer curso de Cálculo, las cuales fueron desarrolladas con el uso de una plataforma interactiva de aprendizaje para el Cálculo 
(PIAC) y un diseño instruccional específico, llamado modelo de unidad de enseñanza (TUM). En tal estudio participaron un total de 102 estudiantes de la Universidad de Colima, quienes matricularon el curso en el 2009 y fueron divididos en dos bloques: uno experimental y otro control del programa de Ingeniería de Telemática (Grupos A y B) y los otros dos del Programa de Bachillerato en Informática (Grupos C y D). La información del desempeño de las y los estudiantes fue recolectada de las actividades de las lecciones, pruebas cortas, tareas y exámenes finales. A partir de los resultados de las observaciones diarias de 140 sesiones, el cuerpo investigador concluyó que las y los usuarios de la plataforma PIAC estaban más motivados y eran más participativos e interactivos entre sí y con cada docente, en comparación con quienes tomaron el curso en el ambiente tradicional (grupos control).

En cuanto al logro académico, quienes participaron de los grupos experimentales completaban satisfactoriamente sus actividades de clase y fueron más atentos durante las lecciones, en comparación con los del control; además, las calificaciones promedio por grupo obtenidas de todas las actividades académicas y la calificación final del curso, en ambos programas, evidenció que la intervención de la tecnología en el diseño instruccional (TUM) se reflejó en el rendimiento académicos del alumnado. Según los autores, los resultados mostraron el potencial del uso de una herramienta tecnológica de calidad y de las unidades de enseñanza en la motivación, que están directamente relacionados con los diferentes procesos cognitivos relevantes para el aprendizaje de la matemática.

El recuento de estas investigaciones en el contexto de la educación superior muestra el interés por comprobar los efectos de la planificación didáctica en los resultados académicos. De hecho, diversos autores motivan al personal docente a desarrollar el diseño instruccional por múltiples razones: genera un mapa de ruta planificado para el proceso de enseñanza y aprendizaje, reduce el riesgo de improvisar acciones y contribuye con la organización y planificación del curso (Bates y Sangrà, 2011; Ertmer \& Newby, 2013; Gil, 2004; Gustafson \& Branch, 2002; Herrera, 2006; Reigeluth, 1983; Reigeluth, 2013; Umaña, 2017). Además, Piskurich (2015) agrega que el estudiantado puede enfrentar un conjunto de situaciones problemáticas, si el docente o la docente no realiza este tipo de planificación.

\section{Procedimiento metodológico}

Esta es una investigación cuasiexperimental en la cual participaron dos grupos que matricularon el curso Cálculo y Álgebra Lineal (CAL) en el segundo semestre del 2013 en la sede de Cartago del Instituto Tecnológico de Costa Rica (TEC). En la modalidad presencial (grupo control) participaron 33 estudiantes y en la modalidad bimodal (grupo experimental) 31, a quienes se les explicó los objetivos de la investigación, la estrategia de trabajo y se les solicitó la firma del consentimiento informado. Al finalizar el semestre, permanecieron 32 estudiantes en el curso presencial y 21 en el bimodal. Para efectos de esta investigación se considera bimodal aquel con lecciones presenciales y virtuales; otras denominaciones son híbrido, semivirtual o blended (en inglés). La estrategia metodológica se desarrolló en dos fases: la planificación del diseño instruccional y la intervención, como se describe a continuación:

\section{Fase de planificación del diseño instruccional}

Previo a impartir el curso, la profesora del curso CAL elaboró el diseño instruccional para ambas modalidades, los cuales fueron revisados por dos expertos en diseño instruccional y en e-learning. Una muestra del resultado de esta planificación didáctica se ilustra en la Tabla 1. 
TABLA 1

Diseño instruccional de una clase sobre series numéricas del curso presencial y del curso bimodal

\begin{tabular}{|c|c|c|c|c|}
\hline \multicolumn{5}{|c|}{ Grupo presencial } \\
\hline $\begin{array}{l}\text { Objetivos de } \\
\text { aprendizaje }\end{array}$ & $\begin{array}{l}\text { Módulos de } \\
\text { contenidos }\end{array}$ & $\begin{array}{l}\text { Actividades de } \\
\text { aprendizaje }\end{array}$ & $\begin{array}{l}\text { Medios y } \\
\text { materiales que } \\
\text { se utilizarán }\end{array}$ & $\begin{array}{l}\text { Evaluación de } \\
\text { los } \\
\text { aprendizajes }\end{array}$ \\
\hline $\begin{array}{l}\text { Aplicar } \\
\text { criterios } \\
\text { para } \\
\text { analizar el } \\
\text { carácter } \\
\text { (convergencia } \\
\text { o } \\
\text { divergencia) } \\
\text { de series } \\
\text { numéricas. }\end{array}$ & $\begin{array}{l}\text { Condición } \\
\text { necesaria } \\
\text { para } \\
\text { convergencia } \\
\text { de series. } \\
\text { Serie } \\
\text { geométrica. } \\
\text { Serie } \\
\text { telescópica. }\end{array}$ & $\begin{array}{l}\text { Clase } \\
\text { magistral con } \\
\text { explicación de } \\
\text { teoria y } \\
\text { ejemplos de } \\
\text { las páginas } 62 \\
\text { a la } 81 \text { del } \\
\text { folleto } \\
\text { Inducción } \\
\text { matemática, } \\
\text { sucesiones y } \\
\text { series. Tarea } \\
\text { modal: } \\
\text { Resolver los } \\
\text { Ejercicios } 10 \text { a } \\
\text { los Ejercicios } \\
12 \text { del folleto } \\
\text { Inducción } \\
\text { matemática, } \\
\text { sucesiones y } \\
\text { series. }\end{array}$ & $\begin{array}{l}\text { Folleto: } \\
\text { Chavarria, J. y } \\
\text { Rodríguez N. } \\
\text { (2013). } \\
\text { Inducción } \\
\text { Matemática } \\
\text { Sucesiones y } \\
\text { Series. TEC } \\
\text { Digital }\end{array}$ & $\begin{array}{l}\text { Quiz } \\
\text { personalizado }\end{array}$ \\
\hline \multicolumn{5}{|l|}{ Grupo bimodal } \\
\hline $\begin{array}{l}\text { Objetivos de } \\
\text { aprendizaje }\end{array}$ & $\begin{array}{l}\text { Módulos de } \\
\text { contenidos }\end{array}$ & $\begin{array}{l}\text { Actividades de } \\
\text { aprendizaje }\end{array}$ & $\begin{array}{l}\text { Medios y } \\
\text { materiales que } \\
\text { se utilizarán }\end{array}$ & $\begin{array}{l}\text { Evaluación de } \\
\text { los } \\
\text { aprendizajes }\end{array}$ \\
\hline $\begin{array}{l}\text { Aplicar } \\
\text { criterios } \\
\text { para } \\
\text { analizar el } \\
\text { carácter } \\
\text { (convergencia } \\
\text { o } \\
\text { divergencia) } \\
\text { de series } \\
\text { numéricas. }\end{array}$ & $\begin{array}{l}\text { Condición } \\
\text { necesaria } \\
\text { para } \\
\text { convergencia } \\
\text { de series. } \\
\text { Serie } \\
\text { geométrica. } \\
\text { Serie } \\
\text { telescópica. }\end{array}$ & $\begin{array}{l}\text { Clase } \\
\text { presencial: } \\
\text { Solución de } \\
\text { ejemplos y } \\
\text { ejercicios. } \\
\text { Clase virtual: } \\
\text { Estudio } \\
\text { independiente } \\
\text { de las páginas } \\
62 \text { a la } 81 \text { del } \\
\text { folleto de } \\
\text { Inducción } \\
\text { matemática, } \\
\text { sucesiones y } \\
\text { series. } \\
\text { Resolver los } \\
\text { Ejercicios } 10 \text { a } \\
\text { los Ejercicios } \\
12 \text { del folleto } \\
\text { de Inducción } \\
\text { matemática, } \\
\text { sucesiones y } \\
\text { series. } \\
\text { Autoevaluación } \\
\text { sobre } \\
\text { contenidos } \\
\text { abarcados } \\
\text { semana } 3 . \text { Se } \\
\text { busca verificar } \\
\text { la } \\
\text { comprensión } \\
\text { de los temas. }\end{array}$ & $\begin{array}{l}\text { Folleto: } \\
\text { Chavarria y } \\
\text { Rodríguez } \\
\text { (2013). } \\
\text { Inducción } \\
\text { Matemática } \\
\text { Sucesiones y } \\
\text { Series. } \\
\text { Autoevaluación: } \\
\text { Classmarker } \\
\text { Facebook o } \\
\text { TEC Digital: } \\
\text { videos, } \\
\text { consultas } \\
\text { virtuales }\end{array}$ & Autoevaluación \\
\hline
\end{tabular}


Como se puede observar, tanto la modalidad presencial como bimodal comparten objetivos y contenidos, las diferencias se presentan en las actividades de aprendizaje y los otros componentes.

\section{Fase de intervención}

La puesta en marcha del curso de CAL se realizó por un periodo de 16 semanas y para la gestión de actividades, recursos y comunicación se utilizó la plataforma tecnológica institucional (o LMS) denominada TEC Digital. En forma complementaria, redes sociales, como Facebook, y otros servicios para compartir recursos educativos, proponer actividades de aprendizaje y de evaluación en ambas modalidades del curso. En esta fase se recolectaron los datos en el siguiente orden cronológico:

1. Semana 1 (julio 2013): Divulgación del proyecto y aplicación del consentimiento informado, el cual fue firmado por el 87,5\% (56 casos) de las y los estudiantes del grupo bimodal y presencial de Cálculo y Álgebra Lineal.

2. Semana 2 (julio 2013): aplicación del pretest de conocimientos básicos de los contenidos del curso de Cálculo y Álgebra Lineal, conformado por una prueba de 10 ítems de selección única, que se calificó en una escala de 0 a 10. El coeficiente de confiabilidad inicial de esta prueba fue de 0,759 y de acuerdo con el análisis del índice de discriminación de los ítems, se eliminó la pregunta No4 por mostrar una correlación negativa, de manera, que la prueba quedó conformada por diez ítems y un $\alpha=0,781$, valor aceptable para efectos de esta investigación.

3. Semana 13 (octubre 2013): aplicación de la técnica de la encuesta usando la escala tipo Likert para medir la percepción que el grupo de estudiantes tiene respecto a los componentes del diseño instruccional entre alumnado de educación universitaria. Antes de la aplicación esta escala fue validada con la técnica de jueces para obtener evidencias asociadas al contenido, específicamente, para determinar el grado de representatividad y pertinencia de las variables que se desean medir. Además, se analizó cualitativamente el instrumento con un grupo focal de estudiantes, para explorar el significado de ciertos vocablos, la comprensión de las instrucciones, errores en el formato y la estimación del tiempo de aplicación.

Luego de aplicada la escala se realizó el análisis de componentes principales para explorar si la configuración factorial del instrumento se ajustaba a la predicha por el modelo teórico del DI. El porcentaje de variancia explicada por los cinco factores fue de 49,40. También se estimó el índice del alfa de Cronbach en la versión final del instrumento, conformado por 33 ítems, cuyo valor fue 0,853 , aceptable para efectos de la investigación. Para una descripción más detallada de la validación de la escala revisar Moreira-Mora y Espinoza-Guzmán (2016).

4. Semana 14 (octubre 2013): Realización de la técnica del grupo de discusión con el propósito de ahondar en las vivencias, acciones y reacciones del alumnado matriculado en los grupos presencial y bimodal de CAL. Para este efecto se construyó una guía de preguntas abiertas relacionadas con cada uno de los componentes del diseño instruccional, el nivel de logro de los objetivos y la modalidad del curso. Específicamente, se les preguntó:

- ¿Qué los llevó a seleccionar la modalidad presencial o bimodal del curso?

- En relación con los contenidos del curso: dificultad, relación entre la teoría-práctica, organización y tiempo de duración.

- En relación con las actividades del curso: actividades favoritas, tiempo asignado, motivación para el aprendizaje por cuenta propia, desarrollo lógico de los temas y formas de comunicación favoritas.

- De los materiales usados en el curso: favoritos y uso de las herramientas tecnológicas. 
- En cuanto la evaluación del curso: en qué momentos se aplicó, en función de los objetivos, usó diferentes formas para la calificación y la realimentación para mejorar su rendimiento.

- Finalmente, se les preguntó cuál fue el nivel de logro de los objetivos del curso y las recomendaciones para mejorar el curso, tanto el presencial como el bimodal.

5. Semana 16: aplicación del postest que fue la misma prueba de conocimientos básicos de los contenidos del curso de CAL usada en el pretest.

6. Semestre: registro a lo largo del semestre de aquellos eventos relevantes del desarrollo del curso mediante una bitácora de observación del docente y aplicación de pruebas parciales y final para asignar la nota final del curso.

\section{ANÁLISIS Y DISCUSIÓN DE RESULTADOS}

El primer análisis realizado fue una comparación de medidas, con la prueba $T$ de student para muestras independientes, del nivel de conocimientos básicos de los contenidos del curso de CAL (dependiente) antes y después del tratamiento, medido con la prueba de selección única. Con la herramienta del SPSS Statistics 19 se estimó el promedio de respuestas correctas. Los resultados fueron similares en ambos grupos tanto en el pretest $(0.16$ y 0,43$)$ como en el postest $(7,00$ y 7,89$)$. En el caso del pretest la diferencia de medias fue de -0.273 , con un nivel de confianza del $95 \%$ y en el postest fue de -0.889 con el mismo nivel de confianza, como se muestra en la Tabla 2.

TABLA 2

Resultados de la prueba de $\mathrm{T}$ en muestras independientes

\begin{tabular}{|c|c|c|c|c|c|c|c|c|c|c|}
\hline & & $\begin{array}{r}\text { Prueb } \\
\text { Leve } \\
\text { para } \\
\text { iguald } \\
\text { variar }\end{array}$ & $\begin{array}{l}\text { a de } \\
\text { ne } \\
\text { la } \\
\text { ad de } \\
\text { azas }\end{array}$ & & & \multicolumn{3}{|c|}{ Prueba $\mathrm{T}$ para la igualdad de medias } & \multirow{2}{*}{\multicolumn{2}{|c|}{$\begin{array}{c}95 \% \text { Intervalo } \\
\text { confianza }\end{array}$}} \\
\hline & & & & & & \multirow{2}{*}{$\begin{array}{l}\text { Sig. } \\
\text { (bilateral) }\end{array}$} & \multirow{2}{*}{$\begin{array}{l}\text { Diferencia } \\
\text { de medias }\end{array}$} & \multirow{2}{*}{$\begin{array}{c}\text { Error típ. } \\
\text { de la } \\
\text { diferencia }\end{array}$} & & \\
\hline & & $\mathrm{F}$ & Sig. & $\mathrm{t}$ & $\mathrm{gl}$ & & & & Inferior & Superior \\
\hline \multirow[t]{2}{*}{$\begin{array}{l}\text { Correctas } \\
\text { pretest }\end{array}$} & $\begin{array}{l}\text { Se } \\
\text { asumen } \\
\text { varianzas } \\
\text { iguales }\end{array}$ & 14.447 & .000 & $\overline{-}$ & 52 & .060 & -.273 & .142 & -.558 & .011 \\
\hline & $\begin{array}{l}\text { No se } \\
\text { asumen } \\
\text { variancias } \\
\text { iguales }\end{array}$ & & & $1 . \overline{78}$ & 32.31 & .084 & -.273 & .154 & -.586 & .039 \\
\hline \multirow[t]{2}{*}{$\begin{array}{l}\text { Correctas } \\
\text { post test }\end{array}$} & $\begin{array}{l}\text { Se } \\
\text { asumen } \\
\text { varianzas } \\
\text { iguales }\end{array}$ & 5.185 & .028 & $\overline{1.36}$ & 41 & .182 & -.889 & .654 & -2.211 & .433 \\
\hline & $\begin{array}{l}\text { No se } \\
\text { asumen } \\
\text { variancias } \\
\text { iguales }\end{array}$ & & & $\overline{1.45}$ & 40.602 & .153 & -.889 & .611 & -2.123 & .345 \\
\hline
\end{tabular}

Como se muestra en los resultados de este modelo estadístico no hay evidencias para rechazar la hipótesis nula de igualdad de promedios, por lo tanto, se puede afirmar que el tratamiento del curso (presencial o bimodal) no se asoció con el rendimiento del estudiantado en la prueba de conocimientos básicos de CAL.

Luego de la aplicación de la escala del diseño instruccional se realizó un análisis integral de cada uno de sus componentes con base en los datos recolectados con las técnicas del grupo de discusión y de la encuesta. 
Específicamente, los resultados de la escala del DI se realizaron con la herramienta del SPSS Statistics 19 y se resumen en la siguiente Figura 1.

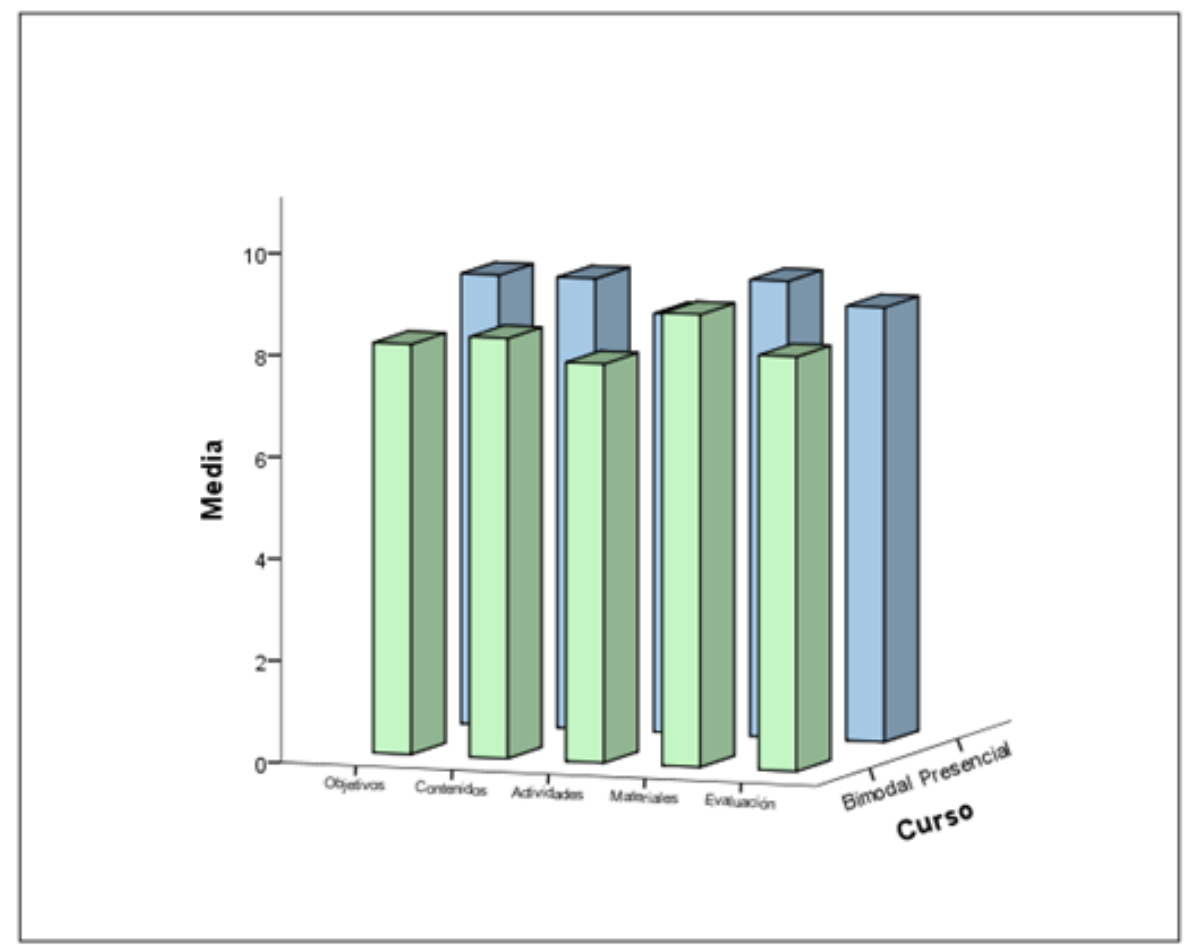

FIGURA 1

Percepción de los estudiantes respecto a los componentes del diseño instruccional del curso Cálculo y Álgebra Lineal en el II semestre 2013, por modalidad del curso

En esta escala tipo Likert los estudiantes calificaban los ítems de cada componente de 1 a 10, a partir de sus percepciones y experiencias en el curso de CAL. Como se muestra en la figura 1, se presentó la tendencia de puntuaciones más altas en el grupo presencial, aunque en general los promedios de cada componente superan la puntuación de 8 , con excepción del componente de actividades en el grupo bimodal que tuvo un promedio de 7,83. Para efectos de esta investigación se consideraron positivos los promedios superiores a 8.

En cuanto al componente de los objetivos del curso los estudiantes de ambas modalidades, que participaron en la técnica del grupo focal, expresaron que el curso "está bien organizado" y que el logro de los objetivos depende, más bien, de su propio esfuerzo, trabajo y organización, especialmente para dedicar tiempo a las actividades fuera de clase. Así mismo, un estudiante del grupo bimodal agrega: "Por mi parte, dedicarle más tiempo porque uno se atiene mucho a que ya entendí, siente que va bien y todo. Entonces no le dedica el tiempo". Estas percepciones positivas fueron respaldadas con los datos recolectados en la escala del DI, donde estudiantes de ambas modalidades asignaron puntuaciones altas (el promedio del grupo presencial fue de 8,83 y el del bimodal fue de 8,05) sobre: el cumplimiento de las expectativas del curso, así como entre la articulación de la teoría con la práctica, el desarrollo de habilidades y su fundamentación con la disciplina matemática.

Sobre los contenidos curriculares, el alumnado de ambas modalidades manifestó en el grupo focal que la estructura y organización son pertinentes y permiten el desarrollo de habilidades de esta disciplina. Al respecto, un estudiante del grupo bimodal indica: "El curso está bien estructurado para personas que en mi caso trabajan, da la oportunidad de entender bien la materia con poco tiempo e incluso sin haber estudiado la materia en la clase presencial uno se podía orientar con facilidad" y uno del grupo presencial añade que el o la docente "siempre llega y nos explica un poco de qué trata un teorema, de qué trata este otro. Digamos 
como un resumen en sí del capítulo, pero muy bien explicado. Entonces después se dedica a desarrollar ejercicios y los va explicando: por el teorema que decía este se resuelve de esta manera. Entonces ya uno puede resolver el ejercicio, siento que en realidad sí está muy bien planteado". Estas afirmaciones concuerdan con la puntuación promedio dada por cada grupo, 8,85 el presencial y 8,25 el bimodal, en este componente del diseño instruccional.

Referente a las actividades de aprendizaje, el estudiantado del grupo presencial contaba con cuatro horas de clases presenciales, donde había exposición de teoría y resolución de ejercicios, en ocasiones las y los estudiantes pasaban a la pizarra a la solución de ejercicios; mientras que la dinámica del grupo experimental era distinta: contaban con dos horas de tutorías presenciales semanales, en donde se presentaba un resumen de los contenidos a estudiarse y luego se realizaban ejercicios involucrando en estos al alumnado, tenían un grupo privado en Facebook para consultas sobre contenidos o ejercicios, además contaban con autoevaluaciones para saber el nivel de comprensión de los contenidos.

En las sesiones virtuales del curso bimodal se aprovechó el aprendizaje autónomo para realizar ejercicios, presentar problemas por resolver y contraejemplos para que las y los estudiantes encontraran el error. También se ofreció consulta en línea por Facebook o por correo, adicional a la consulta presencial. En relación con dichas actividades, consignan que las clases presenciales presentan una secuencia lógica, donde la teoría se desarrolla de forma ordenada, y con los ejemplos y ejercicios, hay espacios para autoevaluarse por sí mismo, así lo argumenta un estudiante del grupo presencial:

Ella al principio siempre repasa, lo vuelve a explicar todo y así uno, sin haber leído el folleto, ya sabe todo lo que le está diciendo y es en la parte de ejemplos en la que uno se pone. Ella es como muy ordenada. Entonces ella dice: punto 1 hacemos esto, número 2 hacemos esto, número 3 esto. Entonces uno lleva el mismo orden que ella lleva en la clase y eso es algo que siempre se ha dado".

Por otro lado, un estudiante del grupo bimodal detalla:

"A mí me gusta que ella escriba el ejercicio en la pizarra y se queda como 5 minutos para que uno lo trate de hacer. Entonces uno ve qué hace y ya después ella lo hace, ahí uno ve si lo hizo bien. Eso me gusta mucho que ella se espera a que uno procese”.

Esta información se comprueba con los datos proporcionados por la escala del DI, donde los estudiantes de ambas modalidades muestran una valoración positiva respecto a las actividades de aprendizaje (presencial 8,22 y bimodal 7,83), pues estas fortalecieron el aprendizaje autónomo, la relación entre teoría y práctica y la interacción entre el o la estudiante.

Los recursos educativos son fundamentales para apoyar los procesos de aprendizaje, en este caso la profesora favoreció el uso de los folletos diseñados para este curso, que contienen teoría, teoremas y otros conceptos matemáticos, así como prácticas, ejercicios resueltos, videos explicativos, contraejemplos y otros materiales que se compartieron por el TEC Digital y el grupo privado de Facebook. Al respecto el estudiantado manifiesta que los materiales siguieron una secuencia lógica, estaban organizados de acuerdo con niveles de dificultad, la materia estaba bien explicada y se entendían con facilidad, además indica un estudiante del grupo bimodal que "el material de apoyo es excelente ya que es muy sencillo y concreto, lo que permite que cualquier persona -incluso sin haber llevado el curso- lo entienda con facilidad" y un estudiante del grupo presencial reafirma la posición de ese estudiante al agregar: "Uno lo lee y ahí viene todo súper explicado en los folletos que ella nos da”.

Los estudiantes del grupo bimodal coinciden en que la red social Facebook es un entorno virtual que les permitió estar actualizados con las actividades propias del curso, así como realizar consultas. Mientras que los estudiantes del grupo presencial indican que a ellos les hubiera servido formar parte de ese conjunto como una forma de comunicación con la profesora y el grupo de compañeros. $\mathrm{Al}$ respecto se presentan extractos de lo expresado por los estudiantes:

Estudiante de curso bimodal: El grupo de Facebook ayuda mucho, por lo menos, a estar al tanto de todo. 
Estudiante de curso presencial: Cuando a uno le chocan todas las horas de consulta con ella, entonces no puedo ir a consulta. Si hubiera un grupo, uno lo puede revisar en la noche o cuando usted tiene libre o algo así, eso sería muy útil...O si usted tiene una duda poder plantearle la consulta y no tener que esperarse hasta el día de consulta y no poder avanzar.

En general, este fue el componente de la escala del DI que obtuvo el promedio más alto en ambos grupos: 8,97 en el presencial y 8,89 en el bimodal, lo que respalda las afirmaciones del alumnado y confirma la importancia de un material adecuado, coherente y preciso para facilitar el aprendizaje del estudiantado.

En relación con la evaluación de los aprendizajes durante el curso se aplicó evaluación diagnóstica, formativa y sumativa. Estudiantes de ambas modalidades realizaron las mismas pruebas parciales y finales; sin embargo, el grupo bimodal contó también con autoevaluaciones donde podían conocer el avance de ellos en ciertos tópicos y foros para la discusión de ejercicios. En las sesiones presenciales, de ambos grupos, se utilizó el método de interrogación para comprobar la comprensión de la materia por parte de los estudiantes y entonces a partir de los resultados aclarar dudas o resolver ejercicios relacionados. En el grupo focal, los estudiantes de ambas modalidades, expresaron que las tareas son las actividades de evaluación que más contribuyeron a reforzar los contenidos. Así en la escala del DI, los estudiantes mostraron una valoración positiva hacia la estrategia de evaluación del curso con puntuación promedio de 8,53 en el presencial y de 8,14 en el bimodal.

Estos resultados demuestran la importancia de una adecuada planificación didáctica del curso para el aprendizaje del estudiantado. En definitiva, resulta un factor clave en los cursos presenciales, bimodales y en línea (Agudelo, 2009; Bates y Sangrà, 201 1; Gil, 2004; Herrera, 2006; Sarsa \& Soler, 2012; Sun, Tsai, Finger, Chen \& Yeh, 2008; Williams, Schrum, Sangrà \& Guárdia, 2004) porque ofrece una guía para estudiantes sobre cómo aprender y sobre cómo desarrollarse (Reigeluth, 2013). Lo relevante, en este sentido, es la estrecha relación entre los componentes del DI, en su estructura y calidad y el efecto que puede generar en el o la estudiante. De hecho, autores como Bates \& Sangrà (2011) y Cabero (2006) definen que la planificación didáctica -y sus componentes- es uno de los factores que contribuyen con la calidad de los procesos educativos. Incluso Van Merriënboer, Clark \& de Croock (como se citó en Frick, Chadha, Watson \& Zlatkovska, 2010), enfatizan que el "diseño [instruccional] inadecuado puede causar problemas de aprendizaje" (p.3).

Finalmente, para responder a la interrogante de si existe relación entre la planificación planteada en el diseño instruccional y el rendimiento académico del estudiantado en el curso de Cálculo y Álgebra Lineal, se estimó el modelo de regresión lineal múltiple, cuyos resultados se muestran en la Tabla 3. En este modelo se incluyó el historial académico del alumnado (promedio simple), que de acuerdo con Dziuban \& Moskal (2011, p. 240) es el más fuerte predictor de éxito en un curso; además de la puntuación en la escala del diseño instruccional (medida DI10), total de materias matriculadas, edad y tratamiento (bimodal o presencial).

TABLA 3

Modelo de regresión lineal múltiple de las variables asociadas a la nota del curso CAL II semestre 2013.

\begin{tabular}{llccccc}
\hline & & \multicolumn{2}{l}{$\begin{array}{l}\text { Coeficientes no } \\
\text { estandarizados }\end{array}$} & $\begin{array}{c}\text { Coeficientes } \\
\text { tipificados }\end{array}$ & & \\
\cline { 2 - 4 } \multicolumn{2}{l}{ Modelo } & $\mathbf{B}$ & Error típ. & Beta & t & Sig. \\
\hline \multicolumn{1}{l}{ (Constante) } & 73.391 & 44.936 & & 1.633 & .111 \\
& Historial TEC & .274 & .213 & .209 & 1.285 & .207 \\
& Total de materias & .558 & .289 & .380 & 1.933 & .061 \\
& Edad & -4.250 & 1.822 & -.416 & -2.332 & .025 \\
& Medida DI10. & 6.382 & 1.954 & .441 & 3.267 & .002 \\
& Tratamiento curso & -3.065 & 3.679 & -.125 & -.833 & .410 \\
\hline
\end{tabular}

a. Variable dependiente: Nota de Cálculo y Álgebra Lineal 
La variancia explicada por el modelo fue del $46 \%$, lo que significa que la variabilidad en el promedio de la nota de CAL es explicada por las cinco variables independientes. Una de las variables con significancia estadística es la edad de los jóvenes que, en promedio y controlando el efecto de las otras, por cada aumento de año tendió a disminuir su promedio del curso en 4,2 puntos (escala de 0 a 100). La edad oscilaba entre los 18 y 26 años, de los cuales el 74\% tenían entre 18 y 20 años, en tanto que la otra cuarta parte se puede calificar con la condición de sobreedad de la o el estudiante.

La segunda variable significativa fue la puntuación dada por el estudiantado al diseño instruccional del curso, cuyo coeficiente estandarizado (.441) fue el más importante de todas las variables. Esto significa que a mayor puntuación en los cinco componentes del diseño (objetivos, materiales didácticos, recursos, actividades de aprendizaje y evaluación), en promedio, el alumnado tiende a obtener 6,3 puntos más en la nota final del curso, controlando el efecto de las otras variables del modelo.

En resumen, con este modelo multivariado se comprueba que existe una relación entre el diseño instruccional y el rendimiento académico del estudiantado de estos dos grupos de CAL, controlado por el efecto del historial académico, la cantidad de materias matriculadas, edad y el tratamiento del curso. Además, este último predictor tampoco resultó significativo en el análisis estadístico de comparación de medias en muestras independientes.

\section{Conclusiones}

A partir de los antecedentes y resultados la principal conclusión de esta investigación es que el diseño instruccional constituyó una especie de columna vertebral del curso CAL, sobre el cual se fundamentaron todas las acciones pedagógicas llevadas a cabo, independientemente de su modalidad, sobre todo, por ser la ruta de trabajo que orientó esta experiencia educativa, la cual resultó positiva en los aprendizajes del estudiantado y se evidenció con el análisis cualitativo y cuantitativo de los datos.

En general, el alumnado percibió positivamente los componentes del diseño instruccional; reconoció la presencia de un plan, el orden, la planificación y el hilo conductor en las acciones pedagógicas que realizó la profesora durante el curso. Interesa resaltar el hallazgo estadístico de que, a mayor puntuación en la percepción del diseño instruccional, en promedio los estudiantes tienden a obtener 6,38 puntos más en la nota final del curso, medida con una escala de 0 a 100. Este hallazgo es significativo, puesto que, conforme el o la docente realice una mejor planificación didáctica, considerando las características de la población meta; organice cada uno de los componentes del diseño instruccional y realice la gestión del curso con el debido seguimiento, retroalimentación, comunicación e interacción; en esa medida el estudiantado percibirá el hilo conductor en el curso y esto repercutirá favorablemente en su rendimiento académico. En este sentido, el carácter sistémico del DI se torna más evidente durante la ejecución del curso (Gustafson \& Branch, 2002; Brown \& Green, 2015; Piskurich, 2015), ya que, todo ajuste que se realice afectará los demás componentes, esto implica la cuidadosa supervisión y seguimiento del aprendizaje del alumnado como un todo y en particular de cada uno de ellos.

Respecto a los componentes del diseño instruccional, se puede resaltar que el estudiantado valoró de manera positiva el cumplimiento de los objetivos del curso y el desarrollo de los contenidos curriculares; tanto por la organización del curso como por la apropiada articulación entre teoría y práctica. La utilización de distintas actividades de aprendizaje (discusiones de ejercicios en clase y en línea, tareas) permitió respetar el estilo y ritmo de estudio de cada estudiante, potenciando el aprendizaje autónomo, lo cual se reforzó con la utilización de folletos propios del curso y videos explicativos. El tener una plataforma de fácil acceso y manipulación como la red social Facebook, favoreció los procesos de enseñanza y aprendizaje pues los estudiantes podían hacer consultas por ese medio y no solo disponer de un horario establecido. Por último, 
en el componente de la evaluación de los aprendizajes el estudiantado indicó que cada tarea fue la actividad que más favoreció la adquisición de conocimiento.

Otro hallazgo relevante se relaciona con el ambiente de aprendizaje. Los resultados permitieron contrarrestar la idea de que los cursos virtuales o bimodales no son buenos, pues ¿cómo aprenderá el o la estudiante si el o la docente no está en clase? Como se confirma en estos resultados, la presencia de la profesora o el profesor no se circunscribe solo a un aula física, sino a las acciones pedagógicas y didácticas que planifique en el diseño instruccional, por lo tanto, esta cobra relevancia en todos los ambientes de aprendizaje, indistintamente de la modalidad virtual, bimodal o presencial. En este caso particular se cae el argumento de que en la bimodalidad no se aprende, ya que la clave del éxito está en propiciar ambientes de aprendizaje con acciones educativas que optimicen los resultados instruccionales deseados.

Los resultados de esta investigación demostraron que existe una relación estadísticamente significativa entre la percepción del estudiantado sobre el diseño instruccional y el rendimiento académico de este en un curso de Cálculo y Álgebra Lineal, quienes percibieron de manera positiva cada uno de los componentes del DI durante su implementación en el curso. Tales hallazgos coinciden con López-Pérez et al. (2011), quienes encontraron que un alto grado de utilidad, motivación y satisfacción percibida por los estudiantes en ambientes bimodales podría llevarlos a tener una actitud más positiva hacia el aprendizaje. Por ello, al igual que Donnelly (2010) consideramos que el aprendizaje en ambientes bimodales no es solo el uso intensivo de actividades con diversas tecnologías; más bien, se trata de una incorporación efectiva de las actividades de aprendizaje e-learning que permitan mejorar, ampliar o transformar las experiencias tradicionales de ambientes presenciales, no se trata de un simple reemplazo. Esta afirmación concuerda con los hallazgos de De la Fuente et al. (2010, p. 811) que afirma: "aunque el rendimiento del alumnado tiene un componente individual importante, no se puede menospreciar el impacto del proceso de [enseñanza y aprendizaje] en él. Un buen diseño y desarrollo del proceso de [enseñanza y aprendizaje] E-A deben llevar consigo un buen producto (mayor satisfacción y rendimiento académico)".

A partir de estos hallazgos, una primera recomendación es brindar más información sobre los cursos bimodales en el Instituto Tecnológico de Costa Rica; debido al desconocimiento de esta modalidad en una universidad caracterizada por el dominio de cursos presenciales. Es recomendable que el alumnado tenga plena conciencia de las implicaciones que esta modalidad acarrea. Además, dada la significancia del diseño instrumental en la planeación de cursos, se recomienda realizar dicho diseño antes de impartir los cursos, así como estar en constante revisión de las actividades y evaluaciones que se proponen, de recursos que se utilizan.

En el futuro, los resultados de esta investigación pueden ser comprobados a mayor escala entre cursos de una universidad. También cabe pensar en buscar si existe correlación entre la percepción de los componentes del diseño instruccional y la evaluación del docente en ese curso específico, que generalmente incluye aspectos de planificación, gestión del curso y evaluación de los aprendizajes.

\section{REFERENCIAS}

Agudelo, M. (2009). Importancia del diseño instruccional en ambientes virtuales de aprendizaje. Nuevas Ideas en Informática Educativa, 5, 118-127. Recuperado de http://cvonline.uaeh.edu.mx/Cursos/DirEducCont/ SeminarioDI/Unidad1/Act15_14_Importancia\%20DI.pdf

Andrade-Aréchiga M., López, G. and López-Morteo, G. (2012). Assessing effectiveness of learning units under the teaching unit model in an undergraduate mathematics course [Evaluar la efectividad de las unidades de aprendizaje bajo el modelo de unidad de enseñanza en un curso de matemáticas de pregrado], Computers \& Education, 59, 594-606. doi:10.1016/j.compedu.2012.03.010

Arjona, M. y Blando, M. (2007). Diseño instruccional, elemento clave en el desarrollo de cursos para ambientes virtuales de aprendizaje. Recuperado de http://bibliotecadigital.conevyt.org.mx/colecciones/documentos/ somece/51.pdf 
Bates, T., and Sangrà, A. (2011). Managing technology in higher education: Strategies for transforming teaching and learning [Gestión de la tecnología en la educación superior: estrategias para transformar la enseñanza y el aprendizaje]. United States of America: John Wiley \& Sons.

Brown, A. H., \& Green, T. D. (2015). The essentials of instructional design: Connecting fundamental principles with process and practice [Lo esencial del diseño instruccional: conectando los principios fundamentales con el proceso y la práctica], United States of America: Routledge.

Cabero, J. (2006). La calidad educativa en el e.Learning: sus bases pedagógicas. Revista de educación, 9(2). Recuperado de http://scielo.isciii.es/pdf/edu/v9s2/original1.pdf

Chavarría, J. y Rodríguez N. (2013). Inducción Matemática Sucesiones y Series. Cartago, Costa Rica: Taller de Publicaciones del ITCR

De la Fuente, J., Martínez, J., Peralta, J. y García, A. (2010). Percepción del proceso de enseñanza-aprendizaje y rendimiento académico en diferentes contextos instruccionales de la educación superior. Psicothema, 22 (4), 806-812

Donnelly, R. (2010). Harmonizing technology with interaction in blended problem-based learning [Armonizando la tecnología con la interacción en el aprendizaje mixto basado en problemas], Computers \& Education, 54, 350 359. doi:10.1016/j.compedu.2009.08.012

Dziuban, C., and Moskal, P. (2011). A course is a course is a course: Factor invariance in student evaluation of online, blended and face-to-face learning environments [Un curso es un curso es un curso: invarianza de factores en la evaluación de estudiantes de entornos de aprendizaje en línea, mixtos y presenciales], Internet and Higher Education, 14(4), 236-241.

Ertmer, P. A., \& Newby, T. J. (2013). Behaviorism, cognitivism, constructivism: Comparing critical features from an instructional design perspective [Conductismo, cognitivismo, constructivismo: Comparación de características críticas desde una perspectiva del diseño instruccional], Performance Improvement Quarterly, 26(2), 43-71.

Frick, T. W., Chadha, R., Watson, C., and Zlatkovska, E. (2010). Improving course evaluations to improve instruction and complex learning in higher education [Mejorando las evaluaciones de los cursos para mejorar la instrucción y el aprendizaje complejo en la educación superior], Educational Technology Research and Development, 58(2), 115-136.

Gil, C. (2004). Modelo de Diseño Instruccional para programas Educativos a Distancia. Revista Perfiles Educativos, XXVI (104), 93-114.

Gustafson, K. L. and Branch, R. M. (2002). What is instructional design? [¿Qué es el diseño instruccional?], Trends and issues in instructional design and technology, 16-25.

Herrera, M. (2006). Consideraciones para el diseño didáctico de ambientes virtuales de aprendizaje: una propuesta basada en las funciones cognitivas del aprendizaje. Revista Iberoamericana de Educación, 38(5), 2.

Landa, P. y Peñalosa, E. (2009). Enseñanza de la psicología clínica conductual, presencial vs en línea: la importancia del diseño instruccional. Acta colombiana de psicología, 12 (1), 109-123.

López-Pérez, M.V., Pérez-López, M.C. and Rodríguez-Ariza, L. (2011). Blended learning in higher education: Students' perceptions and their relation to outcomes. Computers \& Education, 56, 818-826. doi:10.1016/ j.compedu.2010.10.023

Moreira-Mora, T.E. y Espinoza-Guzmán, J. (2016). Initial evidence to validate an instructional design-derived evaluation scale in higher education programs [Evidencias iniciales de validez de una escala para evaluar el diseño instruccional en educación superior]. International Journal of Educational Technology in Higher Education, 13 (11), 1-11. DOI 10.1186/s41239-016-0007-0

Piskurich, G. M. (2015). Rapid instructional design: Learning ID fast and right [Diseño instruccional rápido: ID de aprendizaje rápido y correcto], United States of America: John Wiley \& Sons.

Reigeluth, C. (1983) Instructional-design Theories and Models: An overview of their Current Statu, [Teorías y modelos del diseño instruccional: una visión general de su estado actual], United States of America: Lawrence Erlbaum Associates, Inc. 
Reigeluth, C. (Ed.) (2013). Instructional-design theories and models: A new paradigm of instructional theory [Teorías y modelos del diseño instruccional: Un nuevo paradigma de la teoría instruccional], Vol.II. United States of America: Routledge.

Sarsa, J., and Soler, R. (2012). E-learning quality: Relations and perceptions [Calidad del e-learning: relaciones y percepciones]. International Journal of Information and Communication Technology Education, 8(2), 46-60.

Sun, P. C., Tsai, R. J., Finger, G., Chen, Y. Y., and Yeh, D. (2008). What drives a successful e-Learning? An empirical investigation of the critical factors influencing learner satisfaction [¿Qué impulsa un aprendizaje en línea exitoso? Una investigación empírica de los factores críticos que influyen en la satisfacción del alumno], Computers \& Education, 50(4), 1183-1202.

Umaña, A.C. (2017). La planificación curricular y el diseño curricular de asignaturas en línea. En A.C. Umaña, I. Salas, V. Berrocal. (ed.), Consideraciones para el diseño y oferta de asignaturas en línea (pp. 67-72). San José, Costa Rica: EUNED

Venkatesh, V., Croteau, A. M., and Rabah, J. (2014, January). Perceptions of effectiveness of instructional uses of technology in higher education in an era of Web 2.0 [Percepciones de la efectividad de los usos educativos de la tecnología en la educación superior en la era de la Web 2.0], HICSS '14 Proceedings of the 2014 47th Hawaii International Conference on System Sciences, 110-119.

Van Merriënboer, J. J., Clark, R. E., and De Croock, M. B. (2002). Blueprints for complex learning: The 4C/IDmodel [Blueprints para el aprendizaje complejo: El modelo 4C/ ID]. Educational technology research and development, 50(2), 39-61.

Williams, P., Schrum, L., Sangrà, A., y Guárdia, L. (2004). Fundamentos del diseño técnico-pedagógico. Modelos de diseño instruccional en e-learning. España: Universitat Oberta de Catalunya.

\section{BY-NC-ND}

